\title{
Análisis de los cambios en la profesión contable tras el proceso de implementación de la facturación electrónica en Colombia*
}

\section{Analysis of changes in the accounting profession, \\ after the process of implementing electronic invoicing in Colombia}

Miguel Ángel Laverde Sarmiento* Jairo Alonso Bautista**

Recibido: 17 de febrero de 2020

Revisado: 22 de marzo de 2020

Aprobado: 15 de abril de 2020

* Contador Público, Magister en Ciencias Económicas, Docente de tiempo completo Universidad Santo Tomás, miguellaverde@usantotomas.edu.co

*** Contador Público, Docente de tiempo completo Universidad Santo Tomás, jairobautista@usantotomas.edu.co 


\section{Resumen}

En esta investigación se analiza el efecto de la facturación electrónica en el profesional contable, para identificar los cambios que puede tener en la profesión. Para lograr esto, inicialmente se realiza la revisión de la legislación colombiana en el tema de facturación electrónica, en donde se identifican los principales cambios contables requeridos por ley; posteriormente, mediante encuestas, se establecen los beneficios y dificultades que puede presentar el contador público en Colombia y así analizar las posibles dificultades y ventajas que puedan existir. Luego en el resultado, se identificó que estos cambios pueden afectar a los profesionales y cambiar o eliminar sus dinámicas de trabajo, por lo que estos nuevos cambios pueden marginar a algunos contadores. Adicionalmente, se concluye que es primordial ver este proceso, por una parte, desde una perspectiva a largo plazo, en la que es posible afirmar que la profesión contable se dinamiza debido a la automatización de la información y, por otra, a corto y mediano plazo, pues genera más trabajo técnico.

Palabras clave: cuarta revolución industrial, facturación electrónica, profesión contable, procesos tecnológicos, paradigmas.

Glasificación JEL: M41, A12, A20.

\section{Abstract}

This research analyzes the effect of electronic invoicing on the accounting professional, to identify the changes the profession may have. To achieve this, the review of Colombian legislation on the subject of electronic invoicing is carried out, where the main accounting changes required by law are identified. Subsequently, through surveys, the benefits and difficulties that public accountants in Colombia may have are established, and consequently analyze the possible difficulties and advantages that may occur. The result identifies that these changes can affect professionals and change or eliminate their work dynamics, which is why these new changes can exclude some accountants. In addition, it is concluded that it is of the essence to see this process from the long-term point of view, in which it is possible to state that the accounting profession is revitalized due to the automation of information and, on the other hand, in the short and mediumterm this generates more technical work.

Keywords: Electronic Invoicing, Accounting profession, Technological processes, Paradigms, Fourth Industrial Revolution

JEL Classification: M41, A12, A20. 


\section{Introducción}

La facturación electrónica es un proceso que, en Colombia, se encuentra liderado desde finales del 2015 por la Dirección de Impuestos y Aduanas Nacionales (DIAN) con la pretensión de expandir su aplicación a la mayoría de las empresas del país. Aunque este proceso ha traído problemas tecnológicos y logísticos debido a la complejidad de su ejecución, la DIAN menciona que esta implementación traerá ventajas en el futuro. Estos cambios tecnológicos involucran a los profesionales relacionados con la Contaduría Pública y las Finanzas, los cuales han venido presentando problemas de integración de la tecnología a sus procesos. Por esta razón, mediante esta investigación, se pretende analizar cuál es el efecto de la facturación electrónica en el desarrollo del profesional contable colombiano.

Dado que la facturación electrónica pretende aliviar las tareas operativas para los contadores, así como los procesos administrativos de las empresas, su implementación puede llevar a que los profesionales que ejecutan estas tareas pierdan legitimidad. Por eso, se deben identificar los nuevos procesos que se están llevando a cabo para determinar posibles soluciones (Moll \& Yigitbasioglub, 2019). De igual forma, la facturación electrónica puede ser un paso para, posteriormente, implementar otros tipos de procesos similares como lo son la liquidación de recibos de mercancía de forma automática, el financiamiento de la cadena de suministro y la gestión de administración de gastos, los cuales tienen un impacto en los estados financieros de las compañías (Keifer, 2011). Los cambios generados por la facturación electrónica necesitan una modificación de los paradigmas relacionados con la forma de analizar y abordar los procesos operacionales de las empresas y de los profesionales contables. Cedillo, García, Cárdenas y Bermeo (2018) analizan 1202 investigaciones relacionadas con investigaciones en el tema de facturación electrónica, plataformas y sistemas de notificación, y afirman que aún quedan cuestiones por abordar, aspectos por profundizar e inexistencia de trabajos que analicen este tema en el contexto local. Por lo anterior, esta investigación pretende identificar las problemáticas de la facturación electrónica en el contexto colombiano e identificar posibles soluciones.

Inicialmente, esta investigación se realiza mediante la revisión de la legislación colombiana en el tema de facturación electrónica, en donde se identifican los principales cambios contables requeridos por ley. Posteriormente, a través de encuestas, se establecen los beneficios y dificultades que puede presentar el contador público en Colombia para realizar el proceso de facturación tradicional y electrónica y así contrastar ambos procesos y evidenciar cambios. Finalmente, se analizan las dificultades y las ventajas que puede traer la facturación electrónica a los profesionales contables y se proponen soluciones para las dificultades evidenciadas. 


\section{Marco teórico}

El gobierno electrónico es la forma como el Gobierno se comunica con las personas y las empresas, por medio de las tecnologías de la información (Hai \& Jeong, 2007; Smitha, Thomas, \& Chitharanjan, 2012), y contempla toda comunicación que exista entre gobiernos, personas y clientes como se observa en la tabla 1. El gobierno electrónico también hace referencia al traslado de información desde y hacia el gobierno, por ejemplo, en reportes sobre impuestos, quejas y reclamos, información sobre leyes, reportes estadísticos del país, entre otros. Para analizar cómo está cada país en este tema, la Organización de las Naciones Unidas construyó un índice que mide la prestación de servicios en línea por parte de cada gobierno, en el cual, a 2018, Colombia se encuentra en el puesto 61 entre 193 países, y ocupa el puesto 9 entre los 10 países analizados en América (Department of Economic and Social Affairs, 2018).

Tabla 1. Tipos de relaciones del gobierno en línea

\begin{tabular}{lcccc|} 
& Gobierno & $\begin{array}{c}\text { Empresa } \\
\text { (Business) }\end{array}$ & Cliente & Empleado \\
\hline Gobierno & G2G & G2B & G2G & G2E \\
\hline Empresa(B) & B2G & B2B & B2C & B2E \\
\hline Cliente & G2G & G2B & C2C & N/A \\
\hline
\end{tabular}

Fuente: adaptada de International Federation of Accountants (2002).

Como todo sistema de información el gobierno electrónico tiene sus ventajas y desventajas. Ndou (2004) menciona las siguientes ventajas: reducción en los costos, eficiencia en los ingresos, calidad en los servicios a empresas y clientes, transparencia, reducción de la corrupción, mejora en los procesos contables y mejora en los procesos de toma de decisiones, mientras que, por otro lado, Smitha, Thomas y Chitharanjan (2012) mencionan las siguientes desventajas: grandes inversiones en infraestructura tecnológica, burocracia, exigencias en el desarrollo del capital humano, cambios en la administración y riesgo en la pérdida de información (Zissis \& Lekkas, 2011; Ndou, 2004).

Para potencializar la efectividad y transparencia de este sistema de comunicación, es importante digitalizar y procesar, por medio de canales electrónicos, los diferentes documentos que hacen parte de los insumos de estos procesos (Haag, Born, Kreuzer, \& Bernius, 2013). Uno de estos canales es la factura electrónica la cual se puede definir como una herramienta que incluye la información de un derecho comercial - por medio de un sistema virtual y un cifrado que garantiza la autenticidad (Foryszewski, 2006) - , y cumple con las mismas características del documento físico, en donde, para una parte, es una obligación y, para la otra, un derecho. Adicionalmente, la factura electrónica debe 
cumplir con lineamientos legales dependiendo de su entorno económico (Bartholomew, 2005), por lo que, en el contexto colombiano, se define como un título valor que otorga al poseedor un derecho de cobro, el cual se obtiene de forma virtual, - es decir que no es necesario el documento físico (DIAN, 2019) - y que fue reglamentado por medio del Decreto 2242 de 2015. Por otro lado, Normann (2001) afirma que este canal electrónico hace parte de la desmaterialización de la información, lo cual es una nueva forma de creación de valor.

En Colombia, la facturación electrónica es una cuestión nueva y solamente ha sido implementada por un grupo de prueba, de empresas, mientras que en Europa cada año se envían aproximadamente 30000 millones de facturas y presentan un costo laboral y material de más de $€ 200000$ millones (Association of Corporate Treasurers, 2007). Este aumento en la emisión de facturación se da no solamente por los estímulos legales, sino también por estímulos del mercado (Arendsen \& Van de Wijngaert, 2011). De igual forma, este proceso requiere de mejoras en las prácticas de trabajo y dinamiza el sistema empresarial (Korkman, Storbacka, \& Harald, 2010).

Así, las principales ventajas de esta implementación son la reducción en los costos operativos, la disminución de los errores administrativos y problemas de entrega, la generación de procedimientos contables automáticos, el aumento en la productividad de la empresa, la mejora en las prácticas de manejo administrativo y la mayor eficiencia en la fuerza laboral (Hani, 2001; Korkman, Storbacka, \& Harald, 2010; Lumiaho \& Rämänen, 2011).

En contraste, en la tabla 2 se presentan las desventajas divididas en factores externos e internos:

Tabla 2. Desventajas de la implementación de la facturación electrónica

\section{Factores externos}

La negación del cliente a aceptar la factura electrónica

Diferencia de tecnologías entre proveedores y clientes

Problemas en los proveedores de servicios

Vacíos en temas legales por parte del legislador

Falta de sistemas electrónicos en las pymes

(Karjalainen \& Kemppainen, 2008)

\section{Factores internos}

Dificultades en el uso tecnológico

Costos de implementación

Negaciones a cambiar los sistemas antiguos

Infraestructura insuficiente

Procesos complejos de implementación

Fiabilidad y seguridad del contenido

Fuente: adaptado de The Institute of Financial Operations (2012) 
El gobierno y la facturación electrónicos afectan varios procesos en la empresa, uno de ellos es el proceso contable debido a que la información que transita por estos canales proviene principalmente de la contabilidad. Por lo tanto, se identifica que la informática potencializa la comunicación, la cual es la razón de ser de la profesión contable. Por consiguiente Tua Pereda (2004) y Araujo (1997) aseveran que la contabilidad tiene dentro de sus funciones un enfoque comunicativo, es por esto que la implementación de nuevas herramientas tecnológicas que tengan que ver con el quehacer contable afecta a la profesión, debido a que las transferencias de la información contable pasan de una persona a otra. Según Rendón, Agudelo, y Herrera (2007) el enfoque comunicativo de la contabilidad guarda coherencia con el ambiente tecnológico-informativo que tienen los datos contables hoy en día.

Por otra parte, las empresas deben tener en cuenta que los procesos administrativos no son constantes y se debe estar preparado y atento a los cambios tecnológicos que puedan existir, sobre todo en estos tiempos en donde la tecnología y la forma de comunicación están en constante movimiento. Por eso, en la administración existe la teoría de contingencia (Feldman, 1976; Garengo \& Bititci, 2007) la cual hace referencia a que la administración de un negocio no es rígida y constante, debido a que factores externos e internos pueden afectar a la entidad. Estos factores externos pueden ser la tecnología y la estructura, por lo que, con base en lo anterior, pueden afectar el sistema contable (Chong \& Chong, 1997; Rayburn \& Rayburn, 1991; Wickramasinghe \& Alawattage, 2007).

La inteligencia artificial es la principal involucrada en la forma de comunicarse del gobierno, en el tratamiento de la información contable y financiera y en el estar atentos a los cambios tecnológicos y administrativos de una empresa, debido a que es la base de los cambios tecnológicos. De este modo, la inteligencia artificial se define como la automatización de las actividades relacionadas con el pensamiento humano, como lo son la toma de decisiones, la resolución de problemas y el aprendizaje (Bellman, 1978). Smith (2018), por su parte, la define como un conjunto de programas que replica elementos del comportamiento humano; esto se relaciona con elaboración de software y hardware, también conocidos como Sistemas de Información Interorganizacionales SIIO (IOS, por sus siglas en inglés) los cuales realizan las tareas, organizan procesos y reducen tiempos en las entidades, aportando a la relación entre los diferentes actores de la economía. Los SIIO se empezaron a utilizar en la década de los setenta (Kreuzer, Born, \& Bernius, 2014).

La implementación de estos sistemas afecta a la profesión contable ya que se debe cambiar la forma técnica de procesar y comunicar los datos y, por lo mismo, pueden potencializar el movimiento de la información financiera y contable. Sin embargo, esta automatización reduce las tareas operativas lo cual genera preguntas sobre la legitimidad de la profesión (Moll \& Yigitbasioglub, 2019; Smith, 2018). 
Smith (2018) menciona que la implementación de la inteligencia artificial puede traer los siguientes efectos en la profesión contable:

1. La automatización del trabajo.

2. El cambio hacia un enfoque más avanzado de los profesionales.

3. La organización de los datos traerá una ventaja para el mercado.

4. La auditoría puede ser más precisa debido a que puede obtener los datos de fuentes directas.

5. Información en tiempo real.

6. Se descentraliza la fuente de la información.

7. Revisión continúa de la fiabilidad de la información.

Otra de las dificultades que se observa a nivel formativo es el falso paradigma de que todos los jóvenes son especialistas en informática. Por el contrario, y como lo afirma Stoner (1999), aún no se puede asumir que los estudiantes de contaduría se pueden desenvolver con los aspectos tecnológicos.

\section{Estado del arte}

En la tabla 3 se presentan las principales investigaciones relacionadas con la identificación y el análisis de los factores más relevantes que conlleva la implementación de la facturación electrónica en diferentes países y regiones del mundo.

Luego de esta revisión bibliográfica, se describirá cómo se realizaba el proceso de facturación en Colombia y cómo se va a aplicar posteriormente. En el país, el proceso de facturación tradicional en papel ha sido obligatorio para las empresas que están catalogadas como responsable del impuesto a las ventas, las cuales deben pedir una aprobación de un consecutivo a la DIAN, para que, de esta forma, la entidad pueda controlar qué tantas facturas emiten las empresas. Este procedimiento se realizó hasta el 2015, cuando el Gobierno emitió el Decreto de Facturación Electrónica en el cual se estableció un plan para la implementación de la facturación electrónica con el ánimo de modernizar el sistema transaccional de las ventas, ya que este proceso dejaría de realizarse de manera física para efectuarse, principalmente, a través de medios electrónicos. 
Revista CIFE / ISSN: 0124-3551 e-ISSN: 2248-4914 / Bogotá-Colombia / Vol. 22 N.o 37 / julio-diciembre 2020 / pp. $103-120$

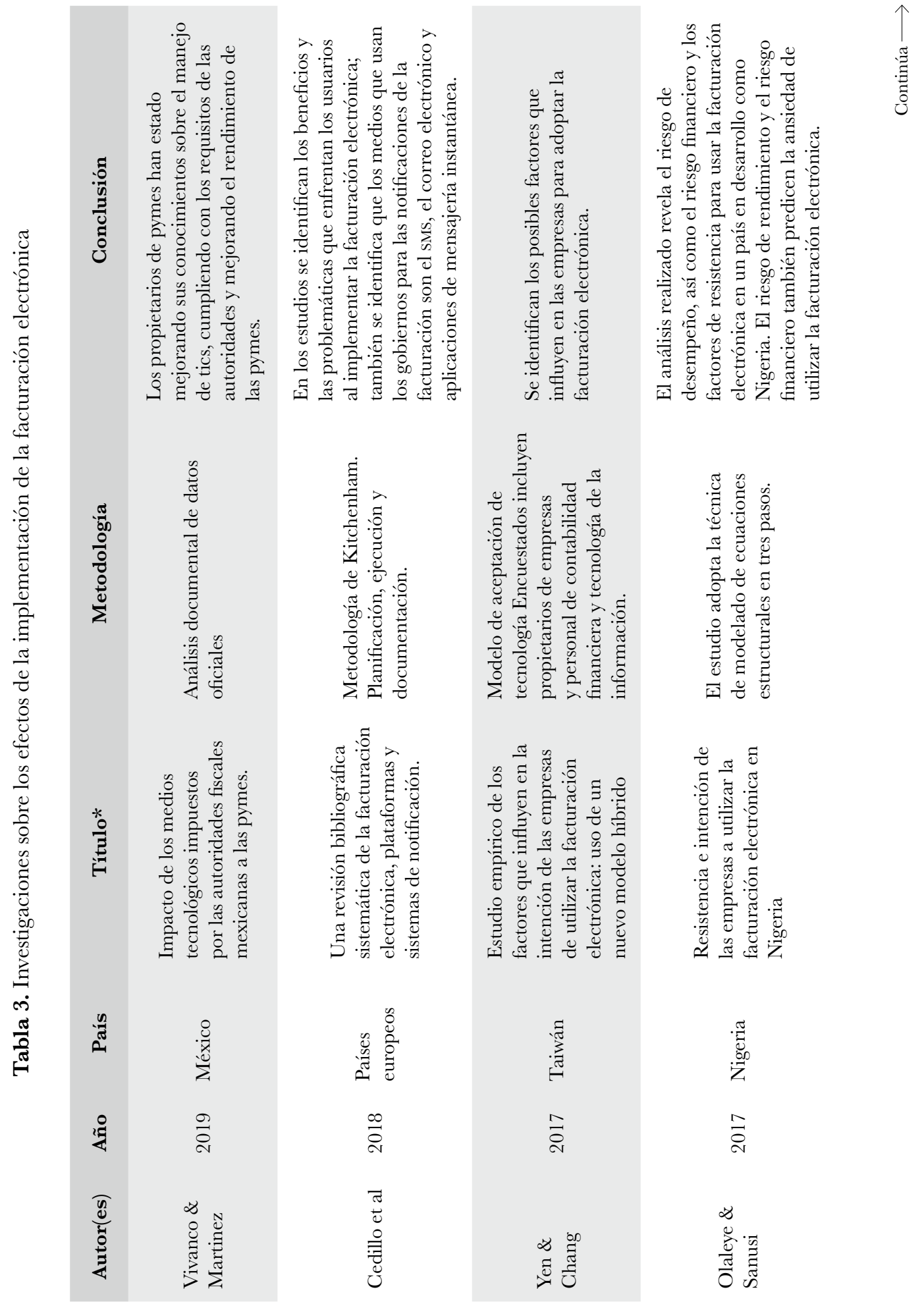




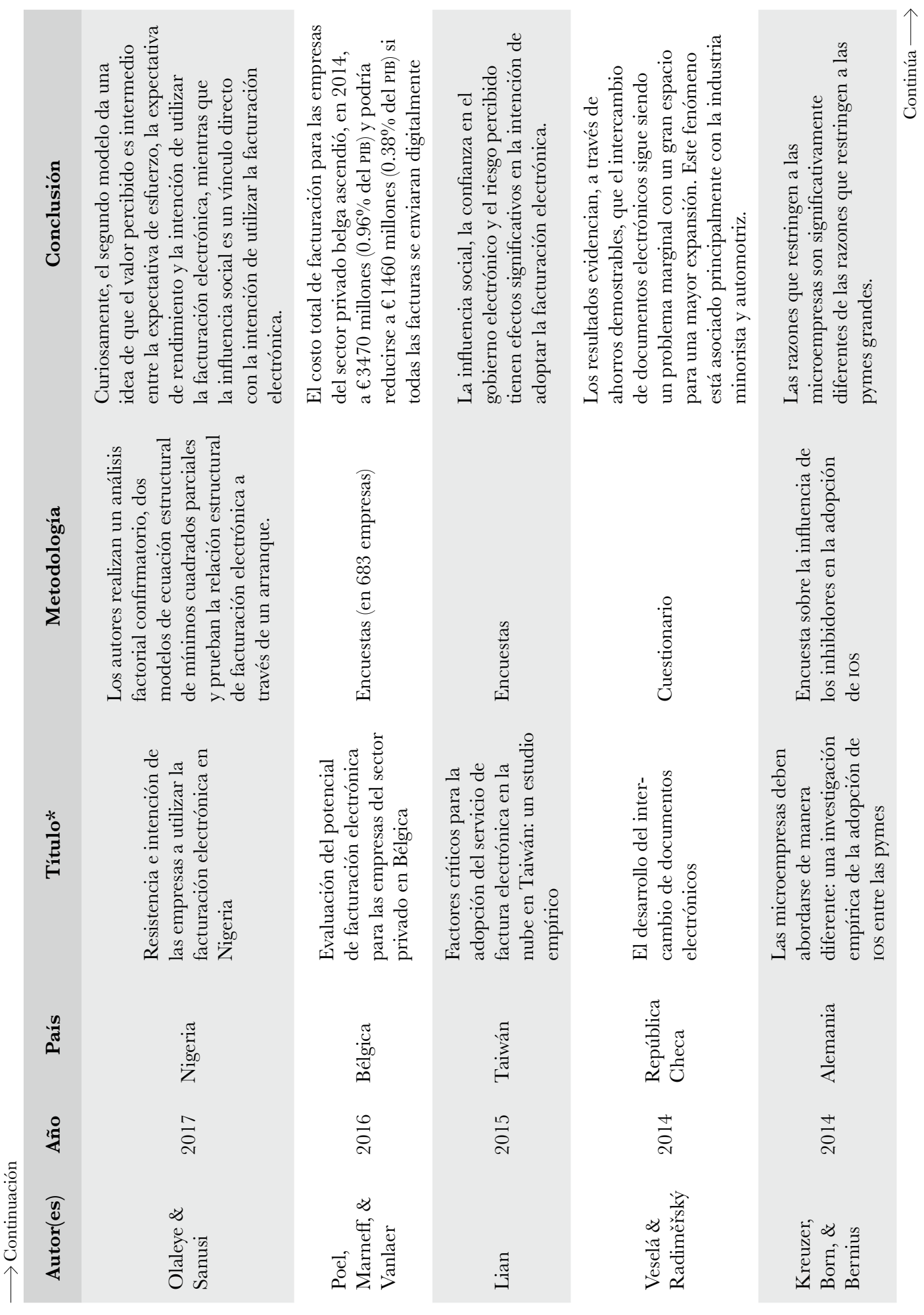


Revista CIFE / ISSN: 0124-3551 e-ISSN: 2248-4914 / Bogotá-Colombia / Vol. 22 N. 37 / julio-diciembre 2020 / pp. $103-120$

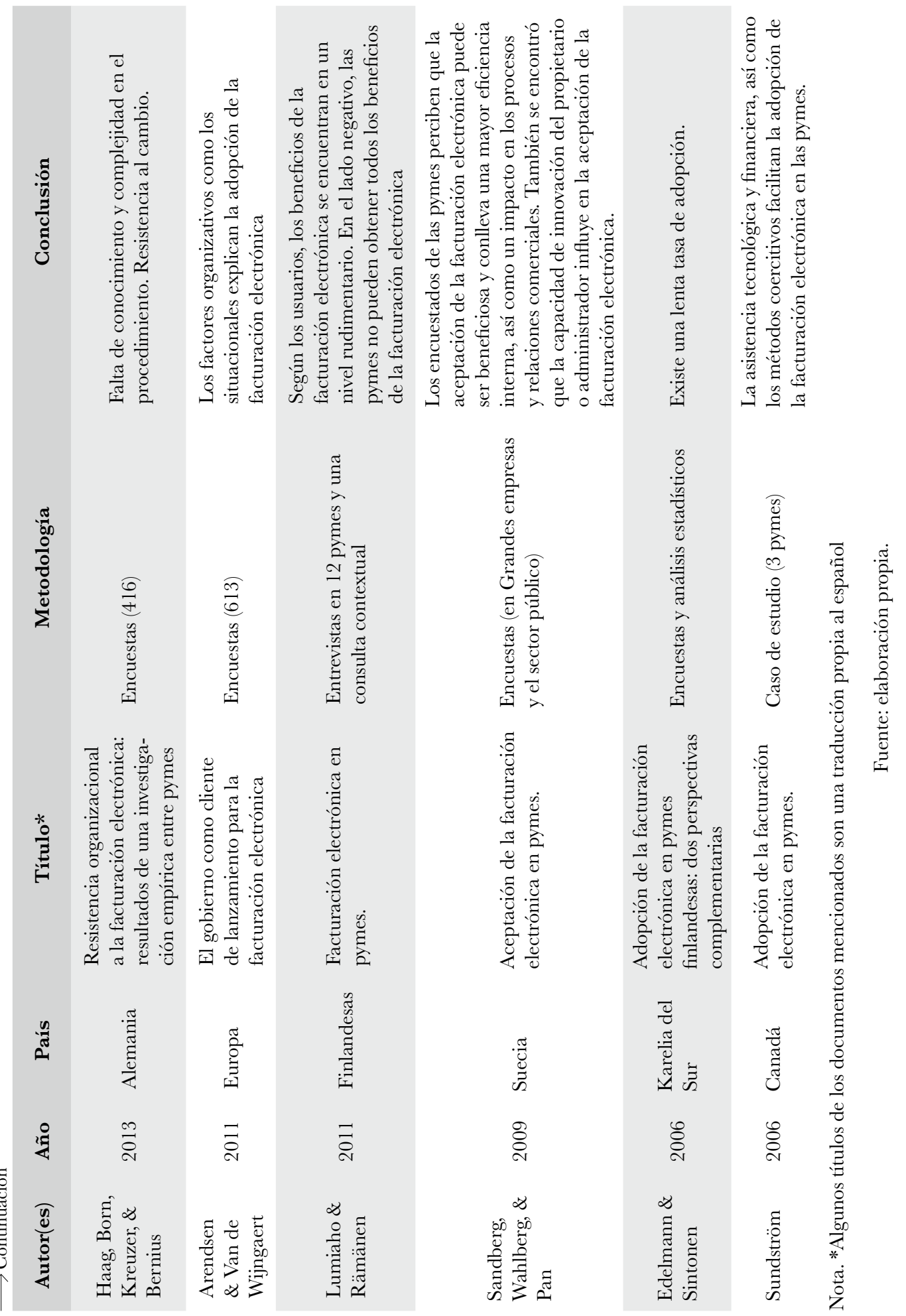


Inicialmente, el Gobierno de Colombia había estipulado que la implementación de este sistema tecnológico se haría a partir del 01 de enero de 2019 y, además, tenía como principal característica que la factura se debía enviar al cliente y, en las 48 horas posteriores, a la DIAN. Más adelante, al identificar que los contribuyentes no habían implementado los sistemas electrónicos para hacer la facturación, el Gobierno decidió ampliar el plazo al 1 de mayo de 2019 y, adicionalmente, cambió el proceso de envío del documento, pues ahora debe enviarse primero a la DIAN y tras la aprobación de este organismo, se podría enviar a los clientes. Por último, el 3 de enero de 2020 surge un proyecto de resolución en el cual se estipula que la implementación iniciará el 1 de febrero de 2020.

El procedimiento para la elaboración y emisión de la facturación tradicional en papel es el siguiente: primero, el comercial cierra el negocio y se estipulaba un contrato; posteriormente, el contador analiza este contrato y realiza la causación correspondiente en el sistema contable, para luego realizar la impresión del documento y entregársela al cliente; finalmente, se hacen las gestiones necesarias para el cobro de cartera. El cambio de la facturación tradicional a la digital trae diferentes transformaciones en este proceso: primero, se debe revisar si el sistema contable cuenta con la herramienta digital adecuada y, posteriormente, se deben hacer correspondientes esto acarrea realizar pruebas de envío a la DIAN y revisar que tanto tarda la respuesta, para enviarle el documento al cliente.

\section{Discusión}

Luego de hacer la revisión conceptual y técnica del proceso de facturación electrónica, se analizarán las complicaciones y ventajas que puede traer esta nueva manera de formalizar los negocios en el país.

La DIAN se ha caracterizado por improvisar en la implementación de diferentes procesos, por lo que la ejecución del sistema de facturación electrónica presenta diferentes complicaciones entre las que se encuentra la incertidumbre por parte de los empresarios; ellos presentan dudas y creencias equivocadas debido a que el Gobierno ha emitido diferentes circulares en las que ha cambiado las reglas y las fechas de la implementación; esto no solamente genera demoras en los procesos, sino que acarrea aumento de costos. Uno de los cambios más significativos ha sido el de validación posterior a validación previa por parte del ente fiscalizador, lo que trajo modificaciones para los diseñadores de los programas informáticos y puede que presente demoras tanto en la respuesta por parte del Gobierno como en el proceso, mientras que y los empresarios pueden perder incluso los negocios pactados. 
Otra complicación es la mala reputación de la DIAN en temas tecnológicos, debido al funcionamiento intermitente de su plataforma llamada MUISCA y a los problemas en los temas de recepción y emisión de información para la presentación de los impuestos, ya que la plataforma presenta demoras en dar respuesta, muchas veces colapsa y deja de funcionar. En este tema específico la plataforma ya cuenta con un aplicativo para elaborar las facturas electrónicas de forma gratuita, sin embargo. ya presenta demoras en dar respuesta y en varias ocasiones no permite generar las facturas.

Dentro de las ventajas se puede considerar la elaboración de este proyecto en torno a la facturación electrónica como algo positivo, debido a que el Gobierno implementa un sistema de control para reducir la evasión, lo cual ayuda a que el recaudo de los impuestos sea mejor. Este tipo de sistemas ya son una tendencia a nivel internacional y Colombia estaba rezagada, por lo tanto, era necesario llevar a cabo esta implementación en la medida en que es un paso inicial para realizar una contabilidad electrónica automática.

Por otra parte, se puede analizar si la implementación de estos procesos puede limitar o aumentar el trabajo de los contadores; es por esto que el hecho de que la DIAN tenga a la mano todas las facturas de las empresas en el país, no implica necesariamente que tengan la contabilidad, pues este es un proceso posterior y algo más complejo - denominado contabilidad electrónica-, que debe hacer dicha entidad y que se encuentra involucrado en los sistemas del gobierno electrónico. Por esta razón, a mediano y corto plazo el trabajo de los contadores aumentará, ya que se deberá realizar doble proceso contable elaborando, primero, la contabilidad en el sistema informático contable de la empresa y, posteriormente, realizando la validación de la información emitida por el organismo de control. Por otro lado, si a futuro se busca aplicar el sistema de contabilidad electrónica como se maneja en México, las empresas tendrían la obligación de emitir información mensual a las entidades encargadas y esto sería más trabajo operativo para los profesionales contables.

Por consiguiente, el cambio de paradigma en este proceso es significativo debido a que inicialmente el proceso era simple donde el contador requería manejar un software o, por lo menos, Excel básico. Ahora, en este nuevo escenario, el profesional requiere un conocimiento más avanzado, debe conocer la plataforma de la DIAN; entender la presentación de alguno de los impuestos para comprender las dinámicas de este sistema y estar atento a los cambios o inconvenientes que allí se pueden presentar, así como tener diferentes conocimientos en tecnología para la elección del proveedor de facturación electrónica, conectividad e integraciones de plataformas para mitigar los errores y hacer el proceso eficiente.

Estos cambios pueden afectar a los profesionales y transformar o eliminar sus dinámicas de trabajo; por eso, los nuevos cambios pueden marginar a contadores debido a que hay 
quienes no manejan las plataformas electrónicas. De allí la importancia de la constante actualización y capacitación por parte de los profesionales. Ahora bien, en el largo plazo este tipo de procesos puede traer ventajas a los contadores debido a que el profesional puede dedicar menos tiempo a la elaboración de informes y envíos a plataformas digitales y para orientarlo al análisis de la información para la toma de decisiones

Por último, si se lograra conectar el proceso que llevará el registro contable del software y automáticamente enviara la información a la DIAN e hiciera la validación para, posteriormente, enviarla al cliente, sería un proceso exitoso. De igual forma debe haber un proceso de sensibilización a los empresarios para que realicen una inversión acertada, ya que la facturación electrónica puede llegar a ser muy dispendiosa y puede generar sanciones al no realizarla correctamente, debido a que es la base de todos los impuestos en el país.

\section{Conclusiones}

La implementación de la facturación electrónica es un proceso que se ha realizado en diferentes países y ha sido de gran ayuda para el control de la evasión y la implementación de la tecnología en los procesos contables y tributarios. Además, esta transición puede traer beneficios u oportunidades para el profesional contable en el país

Han sido varios los cambios que ha realizado el Gobierno colombiano en relación con la facturación electrónica. El proceso inició con la indicación de que a partir del 01 de enero de 2019 se debían enviar las facturas al cliente y, durante las 48 horas posteriores, se le debían enviar a la DiAn. Luego, el plazo estipulado por el Gobierno se amplió al 1 de mayo de 2019 y la factura ya no se enviaría primero al cliente, sino que se le debía enviar a la DIAN y, tras su aprobación, se enviaría a los clientes. Por último, el 3 de enero de 2020, surge un proyecto de resolución en el cual se estipula que la aplicación iniciará el 1 de febrero de 2020 .

La modificación de cualquier dinámica en los procesos contables y tributarios trae diferentes consecuencias en los profesionales que ejecutan estas labores, como ocurre con el cambio en la forma de emitir las facturas. Una de las primeras transformaciones, es la que se debe hacer en el sistema contable, pues debe existir un nuevo módulo que ejecute este proceso, lo que implica hacer pruebas y revisar los procedimientos con el cliente.

El realizar estos nuevos procesos puede afectar directamente a los profesionales contables y cambiar o eliminar la forma de hacer sus trabajos; por eso, las modificaciones, en el corto y mediano plazo, pueden sacar del mercado a contadores que no estén actualizados con temas tecnológicos. Por otro lado, en el largo plazo, pueden traer ventajas 
debido a que los profesionales dedicarán menos tiempo a los procesos técnicos y más al análisis de la información.

Por último, se concluye que existen dos hipótesis que se pueden analizar de lo desarrollado en este documento. La primera es ver el proceso de cambio con una perspectiva a largo plazo y con una visión optimista en la cual se afirma que la profesión contable se dinamiza, debido a que los procesos contables serán automáticos y el contador tendrá que mejorar sus habilidades para analizar la información y presentar informes con un enfoque para la toma de decisiones. En la segunda hipótesis, desde un punto de vista a corto y mediana plazo, se considera que estos procesos de implementación generan más trabajo para los contadores en procesos técnicos; sin embargo, en el contexto colombiano esto significa demoras en las plataformas de información gubernamentales, lo cual genera que los contadores se vean cada vez más enfrascados en procesos que parecieran interminables.

\section{Referencias}

Araújo, J. (1997). Qué es y qué no es contabilidad. Lumina, (2), 101-104. Dor: https:// doi.org/10.30554/lumina.02.1126.1998

Association of Corporate Treasurers. (2007). Corporate action on standards (CAST). Recuperado de http://www.treasurers.org/node/2985

Arendsen, R., \& Van de Wijngaert, L. (2011). Government as a Launching Customer for eInvoicing. En Janssen M., Scholl H. J., Wimmer M. A., Tan Y. (Eds.) Electronic Government. EGOV 2011. Lecture Notes in Computer Science (pp. 122-133). Berlin: Springer. DOI: https://doi.org/10.1007/978-3-642-22878-0_11

Bartholomew, D. (2005). Paper or either? Industry Week, 254(2), 26.

Bellman, R. (1978). An Introduction to Artificial Intelligence: Can Computers Think? San Francisco: Boyd \& Fraser Publishing Company.

Cedillo, P., García, A., Cárdenas, J. D., \& Bermeo, A. (2018, April). A systematic literature review of electronic invoicing, platforms and notification systems. In 2018 International Conference on eDemocracy $\mathcal{E}^{2}$ eGovernment (ICEDEG) (pp. 150-157). IEEE.

Chong, V., \& Chong, K. (1997). Strategic Choises, Environmental Uncerntainty and SBU Performance: A Note on the Intervening Role of Management Accounting Systems. Accounting and Business Research, 27(4), 268-276. DOI: https://doi.org/10.1080/00014 788.1997.9729553 
Department of Economic and Social Affairs, United Nations. (2018). E-Government Survey 2018. Recuperado de: https://publicadministration.un.org/egovkb/Portals/ egovkb/Documents/un/2018-Survey/E-Government\%20Survey\%202018_ FINAL \%20for\%20web.pdf

Dirección de Impuestos y Aduanas Nacionales. (2019). Factura Electrónica. Recuperado de https://www.dian.gov.co/fizcalizacioncontrol/herramienconsulta/FacturaElectronica/Presentacion/Paginas/Queesfacturaelectr $\% \mathrm{C} 3 \%$ B3nica.aspx

Edelmann, J., \& Sintonen, S. (2006). Adoption of electronic invoicing in Finnish SMEs: two complementary perspectives. International Journal of Enterprise Network Management, 1(1), 79-98. DOI: https://doi.org/10.1504/IJENM.2006.010067

El Hani, E. (2001). The e-invoice a legal document? Credit Management, 22-23.

International Federation of Accountants. (2002). E-Business and the Accountant. Recuperado de https://www.icjce.es/images/pdfs/TECNICA/C01\%20-\%20IFAC /C.01.082\%20-\%20ITC\%20-\%20Other/E-Business_Book.PDF

Feldman, D. (1976). A contingency theory of socialization. Administrative Science Quarterly, 21(3), 433-452. DOI: https://doi.org/10.2307/2391853

Foryszewski, S. (2006). The evolution of в2в electronic invoicing. Credit Control, 27 $(4 / 5), 35$.

Garengo, P., \& Bititci, U. (2007). Towards a contingency approach to performance measurement: an empirical study in Scottish sMEs. International fournal of Operations E Production Management, 27(8), 802-825. DOI: https://doi.org/10.1108/01443570710763787

Haag, S., Born, F., Kreuzer, S., \& Bernius, S. (2013). Organizational Resistance To E-Invoicing-Results from an Empirical Investigation among SMEs. En Wimmer M. A., Janssen M., Scholl H. J. (Eds), Electronic Government. EGOV 2013. Lecture Notes in Computer Science (pp. 286-297). Berlin: Springer. DOI: https://doi.org/10.1007/978-3642-40358-3_24

Hai, C., \& Jeong, I. (2007). Fundamental of Development Administration. Selangor, Malasia: Scholar Press.

Karjalainen, K., \& Kemppainen, K. (2008). The involvement of small-and mediumsized enterprises in public procurement: Impact of resource perceptions, electronic systems and enterprise size. Fournal of Purchasing and Supply Management, 14(4), 230240. DOI: https://doi.org/10.1016/j.pursup.2008.08.003 
Revista CIFE / ISSN: 0124-3551 e-ISSN: 2248-4914 / Bogotá-Colombia / Vol. 22 N.o 37 / julio-diciembre 2020 / pp. $103-120$

Keifer, S. (201 1). E-invoicing: The catalyst for financial supply chain efficiencies. Fournal of Payments Strategy ES Systems, 5(1), 38-51.

Korkman, O., Storbacka, K., \& Harald, B. (2010). Practices as markets: Value co-creation in e-invoicing. Australasian Marketing Journal, 18(4), 236-247. DOI: https://doi. org/10.1016/j.ausmj.2010.07.006

Kreuzer, S., Born, F., \& Bernius, S. (2014). Micro-Firms Need to be Addressed Differently an Empirical Investigation of IOS Adoption Among SMEs. Recuperado de https://aisel.aisnet. org $/$ cgi/viewcontent.cgi? article $=1154 \&$ context $=$ amcis 2014

Lian, J. (2015). Critical factors for cloud-based e-invoice service adoption in Taiwan: An empirical study. International Journal of Information Management, 35(1), 98-109. DOI: https://doi.org/10.1016/j.ijinfomgt.2014.10.005

Lumiaho, L., \& Rämänen, J. (2011). Electronic invoicing in SMEs. En A. Marcus, (Ed.) Design, User Experience, and Usability. Theory, Methods, Tools and Practice (pp. 475-484). Berlin: Springer. DOI: https://doi.org/10.1007/978-3-642-21675-6_55

Ministerio de Hacienda y Crédito Público. Decreto 2242 de 2015. Facturación electrónica. Recuperado de https://www.facturador.co/pdf/Decreto_2242_del_24_de_ Noviembre_2015.pdf

Moll, J., \& Yigitbasioglub, O. (2019). The Role of Internet-Related Technologies in Shaping the Work of Accountants: New Directions for Accounting Research. The British Accounting Review, 51(6), 1-20. DOI: http://doi.org/10.1016/j.bar.2019.04.002

Ndou, V. (2004). E-Government for Developing Countries: Opportunities and Challenges. The Electronic Journal of Information Systems in Developing Countries, 18(1), $1-24$.

Normann, R. (2001). Reframing business: When the map changes the landscape. John Wiley \& Sons.

Olaleye, S., \& Sanusi, I. (2017). Companies Resistance and Intention to use Electronic Invoicing in Nigeria. $201714^{\text {th }}$ IEEE India Council International Conference (Indicon) 1-6. DOI: https: / doi. org/10.1109/indicon.2017.8487863

Poel, K., Marneff, W., \& Vanlaer, W. (2016). Assessing the electronic invoicing potential for private sector firms in Belgium. The International Journal of Digital Accounting Research, 16(1), 1-34. DOI: https://doi.org/10.4192/1577-8517-v16_1 
Rayburn, J., \& Rayburn, L. (1991). Contingency theory and the impact of New Accounting Technology in uncertain hospital environments. Accounting Auditing and Accountability fournal, 4(2), 55-75. DOI: https://doi.org/10.1108/09513579110005257

Rendón, N., Agudelo, L., \& Herrera, L. (2007). Enfoque comunicacional de la contabilidad ¿una nueva etapa de la contabilidad? Semestre Económico, 10(19), 125-144.

Sandberg, K., Wahlberg, O., \& Pan, Y. (2009). Acceptance of e-invoicing in smes. En D. Harris (Ed.), Engineering Psychology and Cognitive Ergonomics (pp. 289-296). Berlin: Springer.

Smith, S. (2018). Digitization and Financial Reporting - How Technology Innovation May Drive the Shift toward Continuous Accounting. Accounting and Finance Research, 7(3), 240-250. DOI: https://doi.org/10.5430/afr.v7n3p240

Smitha, K., Thomas, T., \& Chitharanjan, K. (2012). Cloud Based E-Governance System: A Survey. Procedia Engineering, (38), 3816-3826. DOI: https://doi.org/10.1016/j. proeng.2012.06.437

Stoner, G. (1999). IT is part of youth culture, but are accounting undergraduates confident in Iт? Accounting Education, 8(3), 217-237. DOI: https://doi. org/10.1080/096392899330900

Sundström, J. (2006). Adoption of electronic invoicing in sMEs. (Tesis de maestría, Luleå University of Technology). Recuperada de https://www.diva-portal.org/smash/ get/diva2:1019476/FULLTEXT01.pdf

The Institute of Financial Operations. (2012). 2012 Global E-invoicing study: A shift toward e-invoicing ecosystems. Recuperado de https://www.finyear.com/attachment/376412/

Tua Pereda, J. (2004). Evolución y situación actual del pensamiento contable. Revista Internacional Legis de Contabilidad y Auditoría, (20), 43-128.

Veselá, L., \& Radiměřský, M. (2014). The Development of Electronic Document Exchange. Procedia Economics and Finance, 12, 743-751. DoI: https://doi.org/10.1016/ S2212-5671(14)00401-8

Vivanco, J., \& Martinez, M. (2019). Impact of the technological means imposed by the Mexican tax authorities to SMEs. Fournal of Systemics, Cybernetics and Informatics, 17(1), 240-248.

Wickramasinghe, D., \& Alawattage, C. (2007). Management accounting change: Approaches and perspectives. London: Routledge. 
Revista CIFE / ISSN: 0124-3551 e-ISSN: 2248-4914 / Bogotá-Colombia / Vol. 22 N.o 37 / julio-diciembre 2020 / pp. $103-120$

Yen, C., \& Chang, J. (2017). Empirical study of the factors influencing enterprises' intention to use e-invoicing: Use of a novel hybrid model. Fournal of Internet Technology, 18(6), 1285-1296. DOI: https://doi.org/10.6138/JIT.2017.18.6.20150920

Zissis, D., \& Lekkas, D. (2011). Securing E-Government and E-Voting with an open cloud computing architecture. Government Information Quarterly, 28(2), 239-251. DOI: https://doi.org/10.1016/j.giq.2010.05.010 